\title{
MUESTREO DE CANDIDATOS EXTRANJEROS EN LAS ELECCIONES LOCALES ESPANOLAS. NOTA METODOLÓGICA ${ }^{1}$ SAMPLING SPANISH FOREIGN CANDIDATES IN LOCAL ELECTIONS. METHODOLOGICAL NOTE
}

\author{
Óscar Antonio Santacreu Fernández \\ Dpto. Sociología II \\ Universidad de Alicante, España \\ oscar.santacreu@ua.es
}

\begin{abstract}
Resumen
El aumento de la movilidad intraeuropea, potenciado por las sucesivas ampliaciones de la UE, ha puesto en valor la importancia de la participación política de los residentes extranjeros, especialmente en términos de su elegibilidad en las elecciones municipales españolas. En el contexto del proyecto de investigación titulado "La participación política como candidatos de los residentes europeos en España” (Ref. CSO2012-32930), este texto metodológico muestra cómo se ha utilizado un muestreo de tipo onomástico para identificar a los extranjeros que forman parte de las listas de los partidos que se han presentado en las elecciones locales españolas desde el año 2007 al 2015. El trabajo recoge las fases de implementación técnica asociadas al diseño, con la finalidad de determinar la población muestreada.

Palabras clave: Muestreo onomástico, participación política, candidatos, extranjeros, movilidad intra-europea, metodología
\end{abstract}

\section{Abstract}

The increase in intra-European mobility, powered by the successive enlargements of the EU, has higlighted the importance of the political participation

\footnotetext{
${ }^{1}$ Este trabajo se enmarca en el proyecto de investigación "La participación política como candidatos de los residentes europeos en España" (Ref. CSO2012-32930), financiado por la Secretaría de Estado de Investigación, Desarrollo e Innovación del Ministerio de Economía y Competitividad, España.
} 
of foreign residents, particularly in terms of eligibility, in the Spanish municipal elections. In the context of a research project entitled "Political participation as candidates of European residents in Spain" (Ref. CSO2012-32930), this methodological text shows the way we used an onomastic sampling to identify foreigners who are part of the party lists presented in the Spanish local elections, from year 2007 to 2015. This paper covers the technical implementation phases associated with the design, in order to determine the sampled population.

Keywords: onomastic sampling; political participation, foreigners, candidates, intra-european mobility, methodology

\section{Extended abstract}

In the context of the research project entitled "Political participation as candidates of European residents in Spain" (Ref. CSO2012-32930), this methodological text presents a methodological tool that can be used for a better understanding of the process of political mobilization of European foreign residents in Spain. This is a topic of great interest, as it refers to a process associated with increased intra-European mobility, and it determines a process with a great dynamism. The work described in this paper helps us to identify a new elite of foreigners who participate directly as eligible subjects in the Spanish political system. These people shows a great capacity to influence the daily life of the communities in which they are settled.

The methodology described is based on a procedure already used in previous researches funded by the European Union, as the PIONEUR project (Recchi and Favell, 2009) or MOVEACT project, although in the present case this method has been applied in a different way, given that the objectives are also different. We applied an onomastic sampling in order to identify the subjects of interest, that is, candidates in local elections that have one of the following four nationalities: Romanian, Polish, English or German, from the information available on the lists of candidates in the Spanish local elections from 2007 to 2015 in the official newsletters published by the governs of the Spanish provinces. Firstly, we determined first names and most common surnames for each nationality; subsequently we designed a computer algorithm to facilitate the identification of these foreign names and surnames in the lists of candidates. After this, a manual check was applied, and the resulting information was used to improve the procedure interactively.

In the last phase of the procedure, we proceed to the collection and processing of data. For each record, we keep the nationality, the city, the political party, the year, the position held by the candidate in the electoral list, the necessary observations and finally the contact information. Thus we have a valuable information to be used in the later phase of the interviews whose analysis allows to implement the rest of the objectives of the research.

The procedure offered by this paper presents several difficulties that need to be considered. The review of the preliminary results showed a significant number of corrections needed, especially false positives. These corrections were used to provide important feedback to the computer algorithm, by refining 
the dictionaries corresponding to each of the nationalities and also a dictionary of names and surnames which are doubtful and require manual supervision to be assigned to a nationality. Search for information from other public sources, such as newspaper articles or information published on social networks, is necessary to help clarify the nationality of the candidate. However, the use of a specifically programmed algorithm helped us to process easier and faster the identification of foreign candidates among a huge amount of data. Undoubtedly, the use of an automated tool has the virtue of providing a more convenient methodological work when it has an iterative component. Furthermore, this method shows the value and the importance of the review of the results and the feed-back that enriches the methodological phase.

\section{INTRODUCCIÓN}

En el contexto de las sucesivas ampliaciones de la UE, y asociado al incremento de la movilidad intraeuropea, aparece con fuerza el fenómeno sociopolítico de los residentes extranjeros que desarrollan una participación política y cívica muy activa, ya sea fundando asociaciones de diversa índole, afiliándose a partidos políticos nacionales o incluso creándolos de nuevo cuño, movilizando con ello a otros residentes extranjeros.

El artículo 8.B.1 del Tratado Constitutivo de la Comunidad Europea establece que "todo ciudadano de la Unión que resida en un Estado miembro del que no sea nacional tendrá derecho a ser elector y elegible en las elecciones municipales en el Estado miembro en que resida, en las mismas condiciones que los nacionales de dicho Estado". Para desarrollar dicho precepto dentro la legislación española se desarrolló la Ley orgánica 1/1997, de 30 de mayo, de modificación de la Ley Orgánica del Régimen Electoral General. En virtud de dicho desarrollo, la participación política en elecciones locales es un derecho de los ciudadanos de la Unión Europea que residen en otros países, pero también lo es el poder ser elegido. Así, en el artículo segundo de la ley anterior se contempla el derecho de sufragio pasivo en las elecciones municipales que queda con la redacción siguiente:

"1. Sin perjuicio de lo dispuesto en el capítulo II del Título I de esta Ley, son elegibles en las elecciones municipales todas las personas residentes en España que, sin haber adquirido la nacionalidad española: a) Tengan la condición de ciudadanos de la Unión Europea según lo previsto en el párrafo 2 del apartado 1 del artículo 8 del Tratado Constitutivo de la Comunidad Europea, o bien, sean nacionales de países que otorguen a los ciudadanos españoles el derecho de sufragio pasivo en sus elecciones municipales en los términos de un tratado. b) Reúnan los requisitos para ser elegibles exigidos en esta Ley para los españoles. c) No hayan sido desposeídos del derecho de sufragio pasivo en su Estado de origen". 
Tras las ampliaciones de 2004 y 2007, la ciudadanía europea ha sido interpretada principalmente como el derecho a la libre circulación de individuos, permitiendo con ello la migración entre los estados miembros de la Unión Europea. Ejerciendo dicho derecho, desde entonces la población de europeos migrantes ha crecido y se ha diversificado de modo imparable. Las corrientes migratorias internas de europeos forman un colectivo muy heterogéneo: trabajadores manuales (principalmente, pero no sólo, de los países que han accedido recientemente), profesionales con una elevada cualificación, jubilados del norte de Europa en el sur, estudiantes, migrantes que busca una mayor calidad de vida, o incluso parejas mixtas binacionales. No obstante sus diferencias personales, expectativas o planes de vida, estos ciudadanos europeos son en cierto modo los pioneros de la integración europea desde abajo (Recchi y Favell, 2009).

En este contexto se desarrolla el estudio titulado "La participación política como candidatos de los residentes europeos en España" (Ref. CSO201232930). El eje central de este estudio es profundizar en el conocimiento del proceso de movilización política de los residentes extranjeros europeos en España. De forma diacrónica, y usando el análisis narrativo (Penalva, Alaminos, Francés y Santacreu, 2015) a partir de la técnica biográficas de "relatos de vida paralelos", el proyecto pretende describir diferentes procesos de formación de líderes de opinión para establecer los factores personales y contextuales que condicionan la participación política de los ciudadanos de la Unión Europea que viven en un estado miembro diferente de aquél en el que han nacido y crecido. Para ello el proyecto considera los cuatro estados de la Unión Europea que, según los datos de Eurostat (2008) han enviado recientemente más ciudadanos a los demás estados de la Unión Europea. Dado que dos de ellos son nuevos estados miembros (Polonia y Rumania) y los otros dos son de la EU 15 (Gran Bretaña y Alemania), estos cuatro países definen el origen de los migrantes intraeuropeos considerados sobre la base de diferentes culturas políticas de origen: por una parte, democracias consolidadas y, por otra, individuos socializados originariamente en sociedades de influencia comunista. Ello también permite considerar diferentes oportunidades de participación, en el sentido de que ingleses y alemanes han tenido en la práctica cinco elecciones en las que han podido votar y ser elegidos en elecciones locales (1999, 2003, 2007, 2011 y 2015), mientras que Polonia accede a la Unión Europea el 1 de mayo de 2004 y Rumania el 1 de enero de 2007, lo que determina que solamente han tenido oportunidad de participar en tres elecciones. Este hecho permite estratificar la población de candidatos de estas nacionalidades en las elecciones locales según sus oportunidades de participación.

Así, pues, la citada investigación refleja dos grupos bien diferenciados (residentes del Este -polacos y rumanos- y residentes del Oeste -alemanes y britá- 
nicos-) que presentan diferencias económicas (que confiere diferente motivación en cuanto al cambio de residencia y diferentes antecedentes en torno al grado de movilidad internacional, con sus consecuencias derivadas entorno a la dicotomía materialismo/postmaterialismo); diferencias en cuanto a tradición o cultura política (socialismo, liberalismo; y, obviamente, años de pertenencia a la UE) y también una diferente competencia intercultural (que viene determinado principalmente por la cantidad de tiempo de residencia en España, que es mayor en el caso de los alemanes y británicos). En otros estudios se ha analizado la necesidad de estudiar diacrónica y cualitativamente la estrecha relación existente entre vivencias generacionales, socialización política y movilidad política cognitiva (Alaminos, 2008; Alaminos y Penalva, 2012), donde se constatan las limitaciones en cuanto a la aplicación y definición del concepto, que podríamos resumir en una aplicación casi monográfica al ámbito de la movilización de comportamiento electoral, por un lado, y por otro en la carencia de estudios cualitativos que permitan un mejor conocimiento de la relación entre los procesos de movilización política cognitiva y de los procesos de socialización.

El objetivo de este texto, de carácter eminentemente metodológico, se centra en la estrategia orientada a la consecución de un importante objetivo del mencionado estudio, consistente en cuantificar el número de políticos de estas nacionalidades que se han presentado como candidatos en las elecciones locales. En efecto, los datos referidos a la inscripción electoral para votar como electores son bien conocidos, pero no sucede lo mismo con los residentes extranjeros que participan de forma directa en la política española como candidatos.

Además, en este interés por los residentes extranjeros que participan como candidatos en las elecciones locales es fundamental diferenciar los candidatos que se incorporan a partidos políticos tradicionales de ámbito nacional en España, como es el PSOE o el PP por ejemplo, de aquellos otros que se generan desde el entorno social y político de los residentes extranjeros. Consideremos el siguiente ejemplo de un municipio de Alicante llamado San Fulgencio (ver Tabla 1, págs. 478-479).

Los resultados electorales de 2011 fueron del $42,8 \%$ de los votos al PP y 8 concejales; PSOE el 25\% y 4 concejales; APSFU el 21,7\% y 4 concejales; PIPN el 7,59\% y 1 concejal. En términos de gobierno local, el PIPN facilitó su concejal al PP para acceder a la alcaldía y actuó como partido bisagra, dependiendo por tanto de dicho partido la estabilidad del gobierno local.

Por otra parte, dentro de esta dinámica de participación y liderazgo político, son bastante diferentes los perfiles y motivaciones entre los que en esta investigación podríamos denominar residentes extranjeros "empotrados" en listas de partidos de ámbito nacional, que muestran o reconocen una afinidad ideológica con el partido, y los adscritos a partidos de nuevo cuño, que mues- 
Tabla 1. Candidaturas en las elecciones locales de 2011 (San Fulgencio)

P.P. - PARTIDO POPULAR

Titulares

1. Don CARLOS RAMIREZ SANSANO

2. Don PASCUAL SEMPERE MOGICA

3. Don PAULINO HERRERO CIFUENTES

4. Doña MARIA ASUNCION PRIETO CANDELA

5. Doña CONCEPCION CASTELL ESPINOSA

6. Don PEDRO GONZALEZ GARCIA

7. Don BORJA ANTONIO ALONSO AMOROS

8. Don SUSANA ORTUÑO RAMIREZ

9. Don MANUEL GOMEZ REBAGLIATO

10. Doña SHEILA ELIZABETH ANN VALENTINE

11. Don FRANCISCO JOSE ESQUIVA RICO

12. Don ROBERT FREDERICK HARLOW

13. Doña MARIA JESUS MORA MENCHON

14. Don BRIAN SIMONDS

15. Doña ELISABET PEREZ FERRER

16. Don DAVID ALFRED QUINN ENGLISH

17. Doña MARIA CONCEPCION GARCIA BAEZA
P.I.P.N. - PARTIDO INDEPENDIENTE POR LAS NACIONALIDADES

Orden Candidato

1. Don JEFFREY WITOLD WISZNIEWSKI

2. Doña JUANA ROSALIA MORA ALEMANY

3. Doña CARMEN DOLORES SPOLADORE SCHRODER

4. Don DONATO MARIO FALCONE

5. Don FELIX LOPEZ AUñON

6. Doña PIA JANINE SCHRODER

7. Doña TERESA JANE FALCONE

8. Don MANUEL COLOMINAS SANTOS

9. Doña BIENVENIDA DEMETRIA AMOROS PARRES

10. Don ELEUTERIO JOSE BALDO MASIA (independiente)

11. Doña SAMANTHA JAYNE HULL

12. Doña ERIKA LUNA LLANOS

13. Don GEORGE DAVID MCGAW

14. Doña CAROLYN JANE SHEDDEN

15. Don GIORGIO MARIO FALCONE

16. Doña TERESA VIVANCOS MOYA

17. Don JESUS MARIA AMOROS MORA Suplentes

1. Doña JEAN JEFFREY 


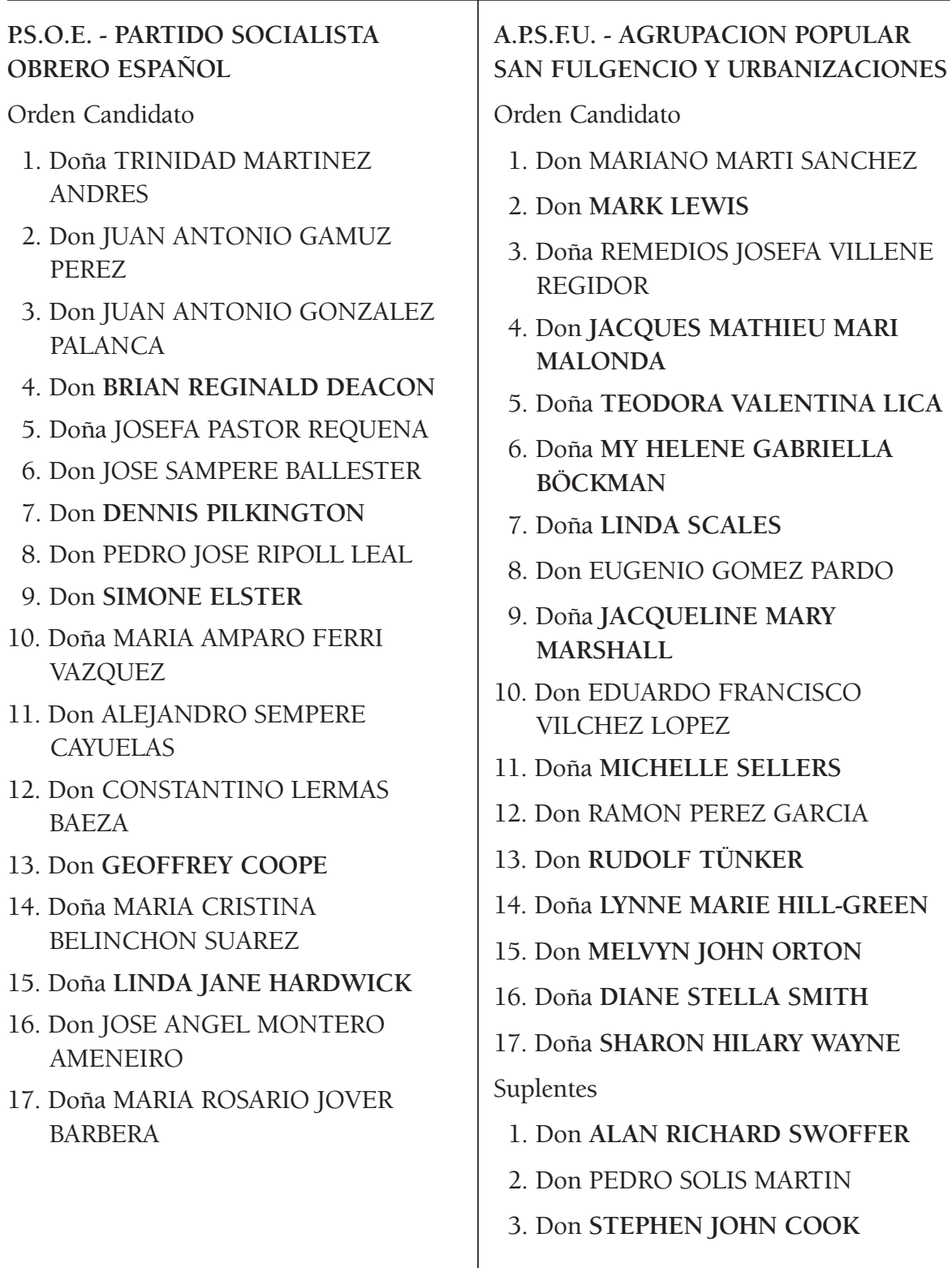

Fuente: BOP de Alicante de 26 de abril de 2011 
tran propiedades muy particulares, tanto en su definición de objetivos e ideología como en su desarrollo organizacional.

El estudio de los candidatos extranjeros en elecciones locales en España, así como la dinámica de nacimiento desarrollo y estabilidad o desaparición de los partidos fundados desde la idea de "extranjería" aporta una información muy relevante sobre las dinámicas de liderazgo, así como de las tensiones entre una visión instrumental de la política y aquellas otras con matriz ideológica.

\section{METODOLOGÍA}

Una vez planteado el objetivo de conocimiento que pretendemos alcanzar, en todo proyecto se abre una etapa de carácter más técnico en la que se debe clarificar de qué manera se puede obtener la información necesaria para el análisis (Francés, Alaminos, Penalva y Santacreu, 2015). Como se ha dicho anteriormente, no existe información sistematizada sobre la inscripción electoral de ciudadanos extranjeros como candidatos en las elecciones que se celebran en la política española. La información existe, dado que los Boletines Oficiales de las provincias en las que se celebran las elecciones locales tienen por norma publicar los listados de las candidaturas de los partidos que se presentan a dichas elecciones. El problema radica en que dichas listas no especifican la nacionalidad de cada uno de los candidatos, por lo que la comprobación manual de dicha nacionalidad se convierte en una ingente tarea cuyo volumen la convierte en poco operativa, a no ser que se aplique un método que permita, en lo posible, automatizar la búsqueda e identificación de candidatos extranjeros en dichos listados.

A partir de los datos disponibles en los listados publicados, y a falta de un mejor indicador, el único criterio viable para discriminar la nacionalidad de los sujetos es la utilización del nombre y/o apellido o apellidos de los individuos como elementos identificativos del país de origen, lo que nos lleva a otra necesidad previa: un criterio que nos permita asignar a un determinado nombre o apellido una probabilidad de pertenecer a una determinada nacionalidad. Para ello utilizaremos el procedimiento de muestreo onomástico descrito en Santacreu, Rother y Braun (2006) y en Braun y Santacreu (2009). Tras este paso previo, dispondremos de un listado de los apellidos y nombres con más probabilidad de pertenecer a cada una de las nacionalidades objetivo en este estudio. El siguiente paso consiste en diseñar un algoritmo que permita el marcado de la información publicada en los listados de los Boletines Oficiales, así como un mecanismo de retroalimentación que permita, mediante la revisión manual de los resultados, afinar el procedimiento para aumentar su eficacia. El procedimiento incluye también la generación de estadísticas con el número de individuos localizados con este método y su asignación a la nacionalidad corres- 
pondiente. Las principales etapas de este procedimiento son, por tanto, las siguientes:

1. Determinación de los nombres y apellidos más frecuentes en cada una de las nacionalidades objetivo, y creación de diccionarios para cada nacionalidad.

2. Diseño de un sistema informático que, de forma automática, detecte y marque los registros que contienen alguno de estos nombres o apellidos en los listados de candidatos que se publican en los Boletines Oficiales de las provincias españolas

3. Comprobación manual del resultado y refinamiento de diccionarios, añadiendo los identificadores detectados manualmente y creando un diccionario de falsos positivos.

4. Reaplicación interactiva del procedimiento a fin de incrementar la fiabilidad y eficacia en la detección de estos candidatos.

5. Recogida y tratamiento de los datos.

A continuación se ofrece una descripción del procedimiento y los primeros resultados obtenidos.

\section{RESULTADOS}

Tal y como hemos descrito anteriormente, el primer paso ha consistido en la determinación de los nombres y apellidos más frecuentes en cada una de las nacionalidades descritas -Gran Bretaña, Alemania, Polonia, Rumanía-. Para ello, además de utilizar los listados que proporcionan algunos centros estadísticos nacionales (INE, 2015), y dada la limitada extensión de estos listados, se ha utilizado la información disponible en listines telefónicos, cuyos registros se han exportado a formato texto (ASCII) con el único fin de permitir su tratamiento estadístico mediante un procedimiento informático automatizado. También se ha utilizado, como veremos, un listado de los nombres y apellidos más frecuentes en España, a fin de eliminar mediante un sistema de lista negra los apellidos o nombres que también son frecuentes entre los españoles, con el objetivo de limitar en lo posible la aparición de falsos positivos.

La información típica recogida en las guías telefónicas constan de los siguientes campos:

- Nombre y apellido(s)

- Dirección

- Código postal

- Población

- Número de teléfono 
Para la extracción se consideraron únicamente los registros correspondientes a particulares, y se desecharon aquellos correspondientes a empresas. Igualmente, se desecharon los campos de Dirección y número de teléfono, dado que no son necesarios en este procedimiento.

A continuación se realizó un análisis de los datos exportados a texto. Así se determinó que el nombre aparecía en el primer campo de cada registro, delimitado por el carácter tabulación (ASCII 9), y que los elementos dentro de cada campo estaban separados por el carácter espacio (ASCII 32). Nombres y apellidos aparecían separados por un carácter visualmente idéntico al espacio, pero distinguible para los algoritmos (el carácter ASCII 160) lo que permitía distinguir entre nombre y apellido (o apellidos). Detectamos que cada país presenta unas características propias; así, muchos registros correspondían a matrimonios e incluían el apellido de cada uno de los cónyuges precedidos del tratamiento, sin incluir el nombre. En el caso alemán, el registro nombre contenía mayoritariamente un apellido y un nombre, si bien en algunos casos aparecían dos individuos en el mismo registro. En el caso inglés, la configuración más frecuente correspondía a un apellido seguido del nombre y, en algunos casos, de un segundo nombre abreviado. A la vista de estas particularidades, se desarrolló un sistema de algoritmos de filtrado ad hoc mediante el cual se extrajeron, de cada registro, dos unidades lingüísticas correspondientes, según el país, a dos apellidos o a un apellido y un nombre. Estas unidades lingüísticas se ordenaron en una lista por país a partir de la cual se calcularon las frecuencias.

Así pues, una vez programado el sistema, compuesto de varios módulos, se recorrió cada uno de los listados proporcionando, para cada país y cada zona geográfica $(z)$, un conjunto de resultados compuesto de las unidades lingüísticas correspondientes a nombres y apellidos:

$$
\Omega_{\mathrm{z}}=\{\text { unidad1,unidad2,unidad3...unidad }\}
$$

Nuestro objetivo consiste en conseguir el subconjunto de unidades lingüísticas "nacionales" con más probabilidades de pertenecer a cada zona geográfica $(z)$ dentro de un país:

$$
\mathrm{A}_{2}=\{\text { unidad1,unidad2... }\}
$$

Ordenando los apellidos de mayor a menor frecuencia, observamos que los apellidos más frecuentes suponían la mayor parte de las frecuencias, por lo que se tomó como elementos del subconjunto $\mathrm{A}_{z}$ aquellas unidades lingüísticas que estaban por encima de un determinado percentil en la zona geográfica $z$. En el Gráfico 1 se observa el caso de Alicante. 
Gráfico 1. Frecuencias absolutas y acumuladas de los apellidos españoles en Alicante

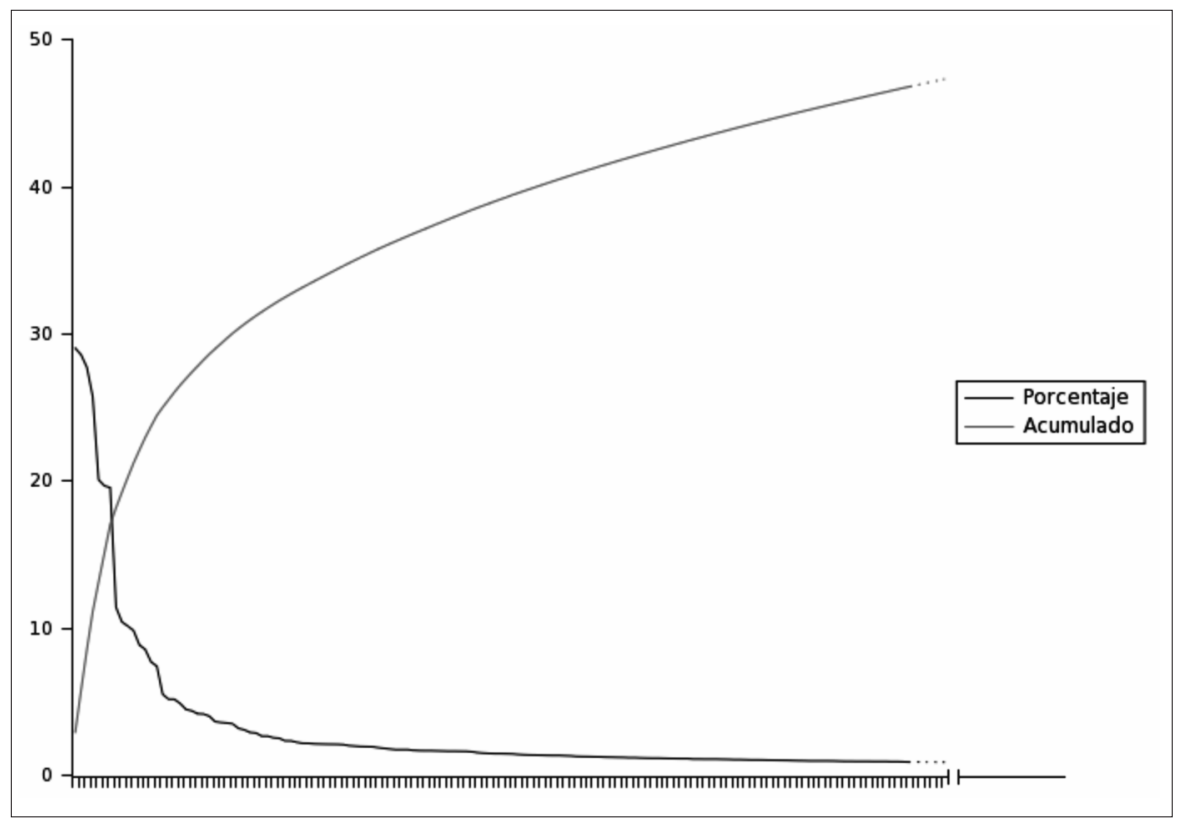

Fuente: elaboración propia

En el gráfico, la línea descendente se refiere al porcentaje que presenta cada apellido, desde el más frecuente al menos frecuente. La línea ascendente representa el porcentaje acumulado. Se observa por tanto que son los apellidos más frecuentes los que, naturalmente, incluyen a un mayor porcentaje de la población, disminuyendo la aportación en términos de población para los apellidos conforme nos alejamos hacia la cola de la distribución.

Por otro lado, el subconjunto $A_{p}$ de cada país resulta de la unión de los subconjuntos correspondientes a cada una de las zonas de dicho país:

$$
A_{p}=\left\{A_{z}{ }^{1} U_{z}{ }^{2} U_{z}{ }^{3} U \ldots A_{z}{ }^{n}\right\}
$$

Seguidamente, el algoritmo calcula la probabilidad para cada uno de los elementos del subconjunto $A_{p}$ partiendo del concepto de frecuencia relativa $f_{\mathrm{r}}=k / n$ siendo $n$ el número de unidades lingüísticas y $k$ las apariciones de la unidad lingüística (regla de Laplace):

$$
\mathrm{P}(\mathrm{A})=(\text { casos favorables }) /(\text { casos posibles })
$$

Se seleccionan entonces aquellas unidades lingüísticas que tienen una mayor probabilidad y se revisa de forma manual el listado a fin de reducir posibles errores. 
Llegados a este punto se ha conseguido una valiosa información: qué unidades lingüísticas (nombres y apellidos) identifican, y con qué probabilidad, la nacionalidad de un individuo cualquiera de la guía telefónica. A continuación se procuró eliminar de los listados de apellidos extranjeros aquellos con alta probabilidad de ser también españoles. Para realizar dicho proceso, el algoritmo eliminó, en primer lugar, los elementos de $A_{o}$ que aparecen en $A_{c}$, es decir:

$$
A_{o}=A_{o}-\left(\begin{array}{ll}
A_{0} & A_{c}
\end{array}\right)
$$

Siendo $A_{o}$ el subconjunto $A_{p}$ del país de origen y $A_{c}$ el subconjunto $A_{p}$ del país de destino (España). Esto quiere decir que si nos encontramos, por ejemplo, con el nombre María como indicador de nacionalidad alemana, dicho nombre sería extraído de $A_{o}$ dado que se encuentra también, por ejemplo, en el subconjunto de España o de Reino Unido.

Este procedimiento sirve para generar un diccionario extenso y optimizado de nombres y apellidos correspondientes a cada una de las nacionalidades que nos interesan. A partir de ahí pasamos al segundo paso, el diseño de un sistema informático que, de forma automática, detecte y marque los registros que contienen alguno de estos nombres o apellidos en los listados de candidatos que se publican en los Boletines Oficiales de las provincias españolas. El algoritmo realiza las siguientes tareas:

1. Abre el archivo y normaliza su contenido (elimina acentos, codifica en UTF8 ${ }^{2}$, convierte a mayúsculas, etc.)

2. Carga en memoria el contenido de los diccionarios de nombres y apellidos correspondientes a cada país, y normaliza su contenido, al igual que en el paso anterior.

3. Establece un contador para cada nacionalidad, a fin de ofrecer estadísticas del procedimiento.

4. Asigna un color a cada nacionalidad para el marcado.

5. Recorre el listado del boletín y procede al marcado y al conteo.

De este modo obtenemos, por un lado, un documento con los candidatos premarcados para su comprobación posterior. Por otro, conseguimos también una valiosa información estadística sobre el número de candidatos de cada nacionalidad detectados en cada una de las circunscripciones.

El algoritmo fue programado en el lenguaje PHP, un lenguaje de programación de uso general, con licencia de software libre, creado originalmente por Rasmus Lerdorf en 1995 (Wikipedia, 2015b). Su nombre es el acrónimo de PHP

${ }^{2}$ UTF-8 (8-bit Unicode Transformation Format) es un formato de codificación de caracteres Unicode e ISO 10646 utilizando símbolos de longitud variable. Actualmente es una de las tres posibilidades de codificación reconocidas por Unicode y lenguajes web, o cuatro en ISO 10646 (Wikipedia, 2015) 
Hypertext Pre-processor, siendo durante muchos años uno de los lenguajes de programación de referencia para el desarrollo web de contenido dinámico, por la fluidez y rapidez que lo caracteriza. la posterior inclusión de una interfaz de línea de comandos ha permitido su utilización en otros entornos, como el caso que nos ocupa. Incorpora una amplia biblioteca nativa de funciones, entre ellas específicas para el manejo de variables de tipo cadena (texto), y proporciona un entorno de programación flexible, potente, manejable, ordenado y estructurado. A modo de ejemplo, la siguiente tabla recoge la función utilizada para normalizar el contenido de los diccionarios y de los listados de candidatos.

Tabla 2. Ejemplo de programación en PHP

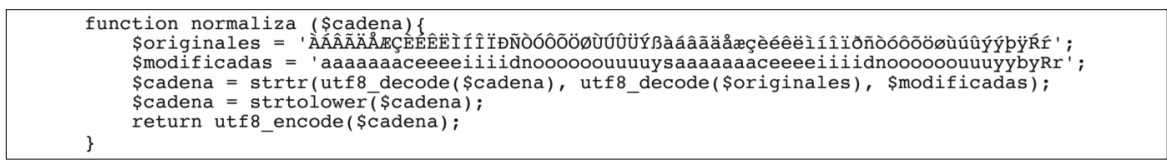

Fuente: elaboración propia

Ofrecemos a continuación, también a modo de ejemplo, las estadísticas de positivos detectados en cinco circunscripciones de Cataluña.

Tabla 3. Estadísticas de candidatos detectados en cinco circunscripciones catalanas

\begin{tabular}{l|l|l|l|l}
\hline Población & Alemanes & Ingleses & Polacos & Rumanos \\
\hline Berga & 1 & 6 & 0 & 3 \\
\hline Valls & 0 & 6 & 1 & 0 \\
\hline El Vendrell & 1 & 9 & 1 & 0 \\
\hline Puigcerdà & 1 & 3 & 0 & 0 \\
\hline Tortosa & 3 & 12 & 0 & 8 \\
\hline$\ldots$ & $\ldots$ & $\ldots$ & $\ldots$ & $\ldots$ \\
\hline
\end{tabular}

Fuente: elaboración propia

Estos pasos no agotan, ni mucho menos, el procedimiento. A continuación viene una de las fases más importantes, consistente en la comprobación manual del resultado y refinamiento de los diccionarios, añadiendo los identificadores detectados manualmente y creando un diccionario de falsos positivos ${ }^{3}$. El objetivo de esta fase no es otro que facilitar la reaplicación interactiva del procedimiento a fin de incrementar la fiabilidad y eficacia en la detección de estos candidatos. Algunas de las tareas realizadas en esta fase fueron:

\footnotetext{
${ }^{3}$ La validación manual ha sido realizada por el investigador Agustín García Ramos.
} 
- Eliminación de duplicidades,

- Comprobación de los nombres de los partidos

- Comprobación de la nacionalidad y ampliación de información sobre candidatos en base a noticias en prensa, redes sociales (Facebook, Linkedin, Twitter...).

- Detección manual de extranjeros no detectados por el procedimiento automático e inclusión de sus datos onomásticos en el algoritmo para permitir futuras detecciones.

- Corrección de apellidos erróneos (por ejemplo, en términos de probabilidad Kuiper no es británico sino holandés, Voltron no es rumano, sino franco-germánico, etc)

En todos los casos se han registrado todos los cambios, tanto en los listados de candidatos como en los diccionarios del algoritmo. En resumen, de un total de 1479 registros detectados por el algorimo en la primera fase, 595 fueron revisados (104 rumanos, 147 polacos, 288 británicos y 56 alemanes). A partir de estos datos revisados se han incluido 136 observaciones catalogadas como falsos positivos únicos en el registro de revisión. A continuación se ofrece un ejemplo de uno de los registros:

"En el documento correspondiente a la Junta Electoral de Zona de Vera (Almería), en la circunscripción electoral de Mojácar, dentro de las candidaturas del partido Somos Mojácar - Para la Gente (SMPLG), existen varios candidatos cuyos apellidos parecen ser británicos y que no han sido marcados, como Adele Helene Land (candidata $n^{\circ} 4$ ), Morgane Valerie Laura Babet (candidata $n^{\circ} 8$ ), o Mark Whitfield (candidato $\left.n^{\circ} 13\right) "$

(Registro de revisión, entrada 42)

Otro producto importante de esta fase, como se ha dicho, es el refinamiento de los diccionarios utilizados por el algoritmo de detección automática. Los datos de los apellidos añadidos en esta primera fase de detección se ofrecen en la siguiente tabla.

Tabla 4: Número de unidades onomásticas (nombres y/o apellidos) añadidas de forma manual a los diccionarios, a partir de la primera revisión manual de los resultados

\begin{tabular}{l|l}
\hline Nacionalidad & Registros añadidos \\
\hline Rumanía & 6 \\
\hline Polonia & 7 \\
\hline Gran Bretaña & 26 \\
\hline Alemania & 3 \\
\hline Apellidos dudosos & 76 \\
\hline
\end{tabular}

Fuente: elaboración propia 
Todo esto nos lleva a la última fase del procedimiento, consistente en la recogida y tratamiento de los datos. Para cada uno de los registros se guardan la nacionalidad, la población, el partido político, el año, el puesto en la lista, las observaciones que sean necesarias e información de contacto para su utilización en la posterior fase de las entrevistas cuyo análisis permita dar cumplimiento al resto de objetivos de la investigación.

\section{DISCUSIÓN}

El procedimiento aquí expuesto no está exento de dificultades. La revisión de los resultados preliminares arrojó un importante número de observaciones, especialmente de falsos positivos, que sirvieron para proporcionar un importante feed-back al procedimiento informático mediante el refinamiento de los diccionarios, tanto los correspondientes a cada una de las nacionalidades como la elaboración de un diccionario de nombres y apellidos dudosos que requieren supervisión manual para ser asignados a una nacionalidad. Como hemos indicado antes, en ocasiones se hace necesaria la búsqueda de información en otras fuentes públicas, como por ejemplo artículos de prensa o información publicada en redes sociales, que ayuden a discernir la nacionalidad del candidato. A pesar de ello, la utilización de un procedimiento informatizado y programado ad-hoc facilita y agiliza la identificación de candidatos extranjeros entre una ingente cantidad de datos.

\section{CONCLUSIONES}

En el contexto del proyecto de investigación titulado "La participación política como candidatos de los residentes europeos en España" (Ref. CSO201232930), este texto metodológico expone una herramienta metodológica que puede utilizarse para profundizar en el conocimiento del proceso de movilización política de los residentes extranjeros europeos en España. Se trata de un tema de gran interés, en tanto que es un proceso asociado al incremento de la movilidad intraeuropea y por tanto de gran dinamismo. El trabajo expuesto en este texto nos ayuda a identificar a una nueva élite de extranjeros que participan de manera directa y como sujetos elegibles en nuestro sistema político con una gran capacidad para influir en la vida cotidiana de las comunidades en las que se establecen. La metodología expuesta está basada en un procedimiento ya probado en investigaciones previas, financiadas por la Unión Europea, como el proyecto PIONEUR ${ }^{4}$ (Recchi y Favell, 2009) o el proyecto

4 "Pioneers of Europe's Integration 'from Below': Mobility and the Emergence of European Identity among National and Foreign Citizens in the EU", investigación financiada por el V Programa Marco de la UE 
MOVEACT ${ }^{5}$, si bien en el caso que nos ocupa se ha aplicado de manera diferente habida cuenta de que los objetivos son también diferentes. Así, se ha utilizado un muestreo de tipo onomástico para identificar a los sujetos de interés, candidatos en las elecciones locales que tienen una de las siguientes cuatro nacionalidades: rumana, polaca, inglesa o alemana, a partir de la información disponible en las listas de candidatos publicadas en los boletines oficiales de las provincias españolas. Para ello se han determinado en primer lugar los nombres y apellidos más frecuentes para cada nacionalidad; seguidamente se ha diseñado un sistema informático que facilite la identificación de estos nombres y apellidos extranjeros en las listas de candidatos, se ha realizado una comprobación manual, y la información resultante se ha utilizado para mejorar el procedimiento de manera interactiva. Finalmente se han comentado brevemente los resultados.

Sin duda, la utilización de herramientas informáticas automatizadas tiene la virtud de facilitar el trabajo de carácter metodológico cuando éste tiene un componente iterativo y es de carácter extenso. Pero además, este procedimiento pone en valor la importancia del factor humano, de la reflexión sociológica, en la revisión de los resultados, así como la importancia de que a partir de esa reflexión se produzca una información de retorno que enriquezca la fase metodológica.

\section{BIBLIOGRAFÍA}

Aja, Eliseo y Díez-Bueso, Laura (2005) "La participación política de los inmigrantes" Puntos de Vista 2

Alaminos, A. (2008) "La movilización política cognitiva y el cambio generacional en Europa" en Tezanos J.F. (ed.) Juventud y Exclusión Social. Madrid. Editorial Sistema.

Alaminos, A.; Penalva, C. (2012) "The Cognitive Mobilization Index: Crises and Political Generations". Sage Open. DOI:10.1177/2158244012440437. Páginas 1-12.

Añón, M. J. (2003) "Nueva ciudadanía y derechos sociales y políticos de los inmigrantes", Gaceta Sindical. Reflexión y Debate 3, 109-136.

Braun, M.; Santacreu, O. (2009) "Appendix A. Methodological notes", en Recchi, E. y Favell, A. (Eds.) Pioneers of European Integration: Citizenship and Mobility in the EU.pp. 241 - 254. Edward Elgar Publishing Limited.

De Asís, Rafael. (2005) La participación política de los inmigrantes. Hacia una nueva generalización de los derechos Instituto de Derechos Humanos Bartolomé de las Casas, http: www. uv.es/CEFD/A.

5 "All Citizens Now: Intra-Eu Mobility and Political Participation of English, Germans, Poles and Romanians in Western and Southern European Cities". Comisión Europea, 2011-2013. 
Durán Muñoz, R.; Martín Martínez, M.; Rodriguez, A. (2007) La participación política de Los extranjeros: estado de la cuestión. Documento de trabajo. JP2007/ 01. Fundación Centro de Estudios Andaluces.

Francés, F; Alaminos, A.; Penalva, C. y Santacreu, O. (2015) El proceso de medición de la realidad social: la investigación a través de encuestas. Cuenca: PYDLOS Ediciones.

Instituto Nacional de Estadística - INE (2015) INEbase / Demografía y población / Padrón. Población por municipios /Apellidos y nombres más frecuentes. Datos procedentes de la Estadística del Padrón Continuo a 1 de enero de 2014 (20 mayo 2015). Consultado en junio 2015 en http://www.ine.es/daco/daco42/nombyapel/ nombyapel.htm

Inglehart, R. (1977). The silent revolution: Changing values and political styles among Western publics. Princeton, NJ: Princeton University Press.

Inglehart, R. (1990). Culture shift in advanced industry society. Princeton, NJ: Princeton University Press.

Méndez, Mónica (2005) "Los derechos políticos de los inmigrantes", en A. Pedreño y M. Pedreño (2005), La Condición inmigrante. Exploraciones e investigaciones desde la región de Murcia, Aula de Debate, Universidad de Murcia pp. 134 y ss. Princeton, NJ: Princeton University Press.

Penalva, C; Alaminos, A.; Francés, F. y Santacreu, O (2015) La investigación cualitativa: técnicas de investigación y análisis con Atlas.ti. Cuenca: PYDLOS Ediciones.

Recchi, E. Y Favell, A. (Eds) (2009) Pioneers of European Integration. Citizenship and Mobility in the EU. Edward Elgar, Cheltenham, UK.

Santacreu, O. (2005) European Union Social Changes: Migrations, Participation and Democracy. Limencop, 2005.

Santacreu, O.; Rother, N.; Braun, M. (2006) Stichprobenziehung für Migrantenpopulationen in fünf Ländern: Eine Darstellung des methodischen Vorgehns im PIONEURProjekt, ZUMA-Nachrichten, pp. 72-88

Wikipedia (2015) Artículo sobre UTF8. Consultado en noviembre 2015 en https:// es.wikipedia.org/wiki/UTF-8

Wikipedia (2015b) Artículo sobre PHP. Consultado en noviembre 2015 en https:// es.wikipedia.org/wiki/PHP

ÓSCAR ANTONIO SANTACREU FERNÁNDEZ es Doctor en Sociología por la Universidad de Alicante. En la actualidad es Profesor Contratado Doctor en el Departamento de Sociología II e investigador del Instituto Interuniversitario de Desarrollo Social y Paz de la Universidad de Alicante. Coordinador académico del Grado en Sociología de la Universidad de Alicante. Sus líneas e investigación son migración y movilidad, participación social, etnocentrismo, integración europea, metodología de la investigación y el desarrollo de tecnologías para la investigación y la docencia. 